\title{
ON PAIRS OF AUTOMORPHISMS OF VON NEUMANN ALGEBRAS
}

\author{
A.B. THAHEEM \\ Department of Mathematics \\ Quaid-i-Azam University \\ Islamabad, PAKISTAN \\ (Received March 18, 1987)
}

ABSTRACT. Let $\alpha, \beta$ be *-automorphisms of a von Neumann algebra $M$ satisfying the operator equation

$$
\alpha+\alpha^{-1}=\beta+\beta^{-1}
$$

In this paper we prove a general decomposition theorem in the non-commuting situation as compared to the usual commuting case (see references) and prove that there exists a central projection $p$ in $M$ such that $\alpha^{2}=\beta^{2}$ on $M p$ and $\alpha^{2}=\beta^{-2}$ on $M(1-p)$.

KEYS WORDS AND PHRASES. Automorphisms, central projections, ideals.

1980 AMS SUBJECT CLASSIFICATION CODE. 46L10; 47C15.

1. INTRODUCTION.

Let $M$ be a von Neumann algebra and $\alpha, \beta$ be *-automorphisms of $M$ satisfying the equation

$$
\alpha+\alpha^{-1}=\beta+\beta^{-1} \text {. }
$$

In this section, we first give a brief description of this equation, (some of) its solutions and applications to get a clear picture of the problem that we intend to resolve in the following sections of this paper. This operator equation if of an active research interest in operator theory since several years. A. Van Daele used this equation in his famous paper on a new approach to the Tomita-Takesaki theory and generalized Hilbert algebras [1] in a special form for unbounded modular operator $\Delta$. In a series of papers the present author has studied this operator equation in detail and has obtained the solutions in various situations (see for instance [2], [3] and [4]). An important question that why this equation after all occurs in a natural way has been considered in [5] (see also [6]). There a bounded map $\psi$ has been associated to a one-parameter group of *-automorphisms $\left\{\alpha_{t}: t \varepsilon R\right\}$ of a von Neumann algebra $M$ by the formula

$$
\psi(x)=\int_{-\infty}^{\infty} \frac{2}{e^{\pi t}+e^{-\pi t}} \alpha_{t}(x) d t, \quad(x \in M) .
$$

It has been shown that determines the group of automorphisms almost completely in the sense that if $\psi_{\alpha}$ and $\psi_{\beta}$ are the maps associated to the one-parameter groups of 
*-automorphisms $\left\{\alpha_{t}: t \varepsilon R\right\}$ and $\left\{\beta_{t}: t \varepsilon R\right\}$ respectively, then $\psi_{\alpha}=\psi_{\beta}$ implies that $\alpha_{t}+\alpha_{-t}=\beta_{t}+\beta_{-t}$ for all $t \varepsilon R$. The study of the equations of this type has led to important applications in Tomita-Takesaki theory. Haagerup and 01sen [7] have considered the mappings $\left\{\alpha_{t}+\alpha_{-t}: t \varepsilon R\right\}$ as a substitute to the modular operator $\Delta$ to develop the Tomita-Takesaki theory for Jordan algebras.

As regards the solutions of the equation $\alpha_{t}+\alpha_{-t}=\beta_{t}+\beta_{-t}$, it has been shown in [4] (see also [8]) using Arveson's theory of spectral subspaces [9] (see also [10]) that $M$ admits a central decomposition such that $\alpha_{t}=\beta_{t}$ on $M p$ and $\alpha_{t}=\beta_{-t}$ on $M(1-p)$, $(t \varepsilon R$ ) for a central projection $p \varepsilon M$. A similar result for derivations has also been obtained. It has been shown that if $\delta_{1}$ and $\delta_{2}$ are the generators of $\left\{\alpha_{t}: t \in R\right\}$ and $\left\{\beta_{t}: t \in R\right\}$ respectively and $\delta_{1}{ }^{2}=\delta_{2}{ }^{2}$ then there exists a central projection $\mathrm{p}$ in $M$ such that $\delta_{1}(\mathrm{p})=\delta_{2}(\mathrm{p})=0$ and $\delta_{1}=\delta_{2}$ on $M p$ and $\delta_{1}=-\delta_{2}$ on $M(1-p)$. In case of factors these results become more interesting (see [4] for details).

The general form of the equation $\alpha+\alpha^{-1}=\beta+\beta^{-1}$ for any pair of automorphisms $\alpha, \beta$ of a von Neumann algebra was considered in [2]. It was shown there (see also [3]) that if $\alpha$ and $\beta$ commute then there exists a central projection $p \varepsilon$ such that $\alpha=\beta$ on $M p$ and $\alpha=\beta^{-1}$ on $M(1-p)$. This decomposition has played an important role in the geometric interpretation of the Tomita-Takesaki theory [11]. The commutativity assumption of $\alpha$ and $\beta$ has been very important to the solution of this equation. In some situations, it has been shown that the equation $\alpha+\alpha^{-1}=\beta+\beta^{-1}$ already implies the commutativity of $\alpha$ and $\beta$ and thus a decomposition of $M$ can be obtained in a simpler way (for more details, see [2]).

In this paper we consider the equation $\alpha+\alpha^{-1}=\beta+\beta^{-1}$ in a general form and without assuming the commutativity of $\alpha$ and $\beta$. In section 2 of this paper we construct an example of automorphisms $\alpha$ and $\beta$ satisfying the equation $\alpha+\alpha^{-1}=\beta+\beta^{-1}$ on a von Neumann algebra $M$ but there is no decomposition for which $\alpha=\beta$ on one part and $\alpha=\beta^{-1}$ on the other part. In section 3, we show, however, that there exists a central projection $p$ in $M$ such that $\alpha^{2}=\beta^{2}$ on Mp and $\alpha^{2}=\beta^{-2}$ on $M(1-p)$. This provides a general solution of the problem for von Neumann algebras.

We believe that the techniques of this paper and out previous papers concerning this problem may contribute to a new and simpler way to study automorphism groups and derivations. In a subsequent paper we intend to use this theory to study a problem on one-parameter groups of automorphisms on a von Neumann algebra.

Recently, Sakai [12] suggested this problem for $C^{\star}$-algebras and thus it remains an unsolved problem.

2. AN EXAMPLE.

Let $M_{2}$ denote the algebra of all $2 \times 2$ complex matrices and consider the von Neumann algebra $M=M_{2}+M_{2}$, the direct sum of two copies of $M_{2}$. Let $u$ and $v$ be two unitaries in $M_{2}$ and define two automorphisms $\alpha$ and $\beta$ on $M$ by

$$
\begin{aligned}
& \alpha(x, y)=(u y u *, x) \\
& \beta(x, y)=(u v * y v u *, v x v *)
\end{aligned}
$$


It is easy to check that $\alpha$ and $\beta$ are indeed *-automorphism of $M$.

We want to have some conditions on $u$ and $v$ such that $\alpha+\alpha^{-1}=\beta+\beta^{-1}$. Now

$$
\begin{aligned}
& \alpha^{-1}(x, y)=\left(y, u^{*} x u\right) \\
& \beta^{-1}(x, y)=\left(v^{*} y v, v u^{*} x u v^{*}\right) .
\end{aligned}
$$

So we must have

$$
\begin{aligned}
u_{y u}^{*}+y & =u v^{*} y v u^{*}+v^{\star} y v \\
x+u^{*} x u & =v x v^{\star}+v u^{\star} x u v^{\star} \\
& =v\left(x+u^{\star} x u\right) v^{\star}
\end{aligned}
$$

If we set $x=u$ in equation (2.2) we obtain $2 u=2 v u v *$ and hence $u v=v u$.

We see from equation (2.2) that $v\left(x+u^{*} x u\right)=\left(x+u^{*} x u\right) v$ for any $x \in M_{2}$. Also using the fact that $u v=v u$ (and $u v^{*}=v^{*} u$ ), we see from equation (2.1) that $v\left(u^{*} u^{*}+y\right)=\left(u_{y}^{*}+y\right) v$ for any $y \varepsilon M_{2}$. Thus, we see that the two equations give the same commutability relations.

Without loss of generality we assume that us is diagonal. So

$$
\mathbf{u}=\left(\begin{array}{ll}
\lambda & 0 \\
0 & \mu
\end{array}\right), \lambda \mu \varepsilon \phi \text {. }
$$

Now we want to determine the set

$$
v=x+u^{\star} x u: x \in M_{2}
$$

with the condition that $v$ commutes with all the operators from this set.

$$
\text { If } \begin{aligned}
x & =\left(\begin{array}{ll}
1 & 0 \\
0 & 0
\end{array}\right) \text { then } x+u^{*} x u=\left(\begin{array}{ll}
2 & 0 \\
0 & 0
\end{array}\right) \\
x & =\left(\begin{array}{ll}
0 & 0 \\
0 & 1
\end{array}\right) \text { then } x+u^{*} x u=\left(\begin{array}{ll}
0 & 0 \\
0 & 2
\end{array}\right) \\
x & =\left(\begin{array}{ll}
0 & 1 \\
0 & 0
\end{array}\right) \quad \text { then } x+u^{*} x u=\left(\begin{array}{ll}
0 & 1+\bar{\lambda} \mu \\
0 & 0
\end{array}\right) \\
x & =\left(\begin{array}{ll}
0 & 0 \\
1 & 0
\end{array}\right) \quad \text { then } x+u^{*} x u=\left(\begin{array}{ll}
0 & 0 \\
1+\lambda \bar{\mu} & 0
\end{array}\right)
\end{aligned}
$$

If $\bar{\lambda} \mu \neq 0$ then $v=M_{2}$ and $v$ would be a scalar operator which means that $\alpha=\beta$. So we assume that $\bar{\lambda} \mu=-1$ or equivalently $\mu=-\lambda$. We can assume that $\lambda=1$ and $\mu=-1$ because changing $u$ by a scalar does not affect the automorphism. So if $u=\left(\begin{array}{rr}1 & 0 \\ 0 & -1\end{array}\right)$ then $u=u^{*}$ and $u(x+u x u) F(x+u x u) u=x+u x u$. Thus $x+u x u$ is a diagonal matrix.

Now, if we choose $v$ to be a diagonal matrix, we will have all our conditions and 
$\alpha+\alpha^{-1}=\beta+\beta^{-1}$. If $v=\left(\begin{array}{ll}\lambda & 0 \\ 0 & \mu\end{array}\right)$ with $\lambda \neq \mu$ so that $v$ is not a scalar and hence $\alpha \neq \beta$. When $\lambda \neq-\mu$ then $v^{*}$ is not a scalar and hence $\alpha \neq \beta^{-1}$.

Thus, there is not central decomposition of $M$ such that $\alpha=\beta$ on one part and $\alpha=\beta^{-1}$ on the other part.

It is easy to check that in the example above

$$
\begin{aligned}
& \alpha^{2}(\mathrm{x}, \mathrm{y})=\left(\mathrm{uxu}^{*}, \mathrm{uyu}^{*}\right) \\
& \beta^{2}(x, y)=\beta\left(u v^{*} y_{v u}^{*}, v_{x u}^{*}\right) \\
& =\left(u v^{\star}\left(v x v^{\star}\right) v^{*}, v u v^{*} \mathrm{yvu}^{\star} \mathrm{v}^{\star}\right) \\
& =\left(\mathrm{uxu}^{*}, \mathrm{uyu} \mathrm{u}^{*}\right)
\end{aligned}
$$

so that $\alpha^{2}=\beta^{2}$. This is the situation that we intend to obtain in general in the next section.

3. CENTRAL DECOMPOSITION.

In this section we prove that if $\alpha, \beta$ are *-automorphisms of a von Neumann algebra $M$ and $\alpha+\alpha^{-1}=\beta+\beta^{-1}$, then $M$ can be decomposed by a central projection such that $\alpha^{2}=\beta^{2}$ on one part and $\alpha^{2}=\beta^{-2}$ on the other part. If $M$ is a factor then either $\alpha^{2}=\beta^{2}$ or $\alpha^{2}=\beta^{-2}$.

We begin with the following

PROPOSITION 3.1. Let $M$ be a von Neumann algebra which is the direct sum of type I factors and $\alpha, \beta$ be $*$-automorphisms of $M$ satisfying the equation $\alpha+\alpha^{-1}=\beta+\beta^{-1}$. Then there exists a central projection $p$ in $M$ (invariant under $\alpha$ and $\beta$ ) such that $\alpha^{2}=\beta^{2}$ on $M p$ and $\alpha^{2}=\beta^{-2}$ on $M(1-p)$.

PROOF. We may assume that $M=\sum_{i \varepsilon I I} \oplus M_{1}$, where $M_{i}$ are type $I$ factors. The center $Z$ of $M$ can be identified with $\ell_{\infty}$ (II). To each $i \varepsilon$ II, we can associate a central minimal projection $e_{i}$ which corresponds to an element of $l_{\infty}$ (II) which is 1 for 1 and 0 elsewhere. Each automorphism leaves the center invariant and the central minimal projections are transformed into the central minimal projections.

Now take a central minimal projection $e$ in $M$. Then there are two possibilities: Either $\alpha(e)=\alpha^{-1}(e)$ or $\alpha(e) \neq \alpha^{-1}(e)$. If $\alpha(e)=\alpha^{-1}(e)$ then $\alpha^{2}(e)=e$. Taking square of the equation $\alpha+\alpha^{-1}=\beta+\beta^{-1}$, we get that $\alpha^{2}+\alpha^{-2}=\beta^{2}+\beta^{-2}$; hence $\beta^{2}(e)+\beta^{-2}(e)=2 e$ and by [2] $\beta^{2}(e)=e$. Thus $\alpha^{2}=\beta^{2}$ or $\alpha^{2}=\beta^{-2}$ on a type I factor Me.

Next assume that $\alpha(e) \neq \alpha^{-1}(e)$. Then we must have $\beta(e) \neq \beta^{-1}$ (e) because $B(e)=\beta^{-1}(e)$ implies $\alpha^{2}(e)=e$ or $\alpha(e)=\alpha^{-1}(e)$ by the above considerations. Since $\alpha(e)$ and $\alpha^{-1}$ (e) are both central minimal projections and are distinct, therefore they must be orthogonal. Similarly $\beta(e)$ and $\beta^{-1}(e)$ are orthogonal.

Now $\alpha(e)+\alpha^{-1}(e)=\beta(e)+\beta^{-1}(e)$. By minimality condition, $\alpha(e)=\beta(e)$ or $\alpha(e)=\beta^{-1}(e)$. If $\alpha(e)=\beta(e)$ and $x \varepsilon$ Me then $\alpha(x)+\alpha^{-1}(x)=\beta(x)+\beta^{-1}(x)$. Now $\alpha(x) \varepsilon M \alpha(e), \alpha^{-1}(e) \varepsilon M \alpha^{-1}$ (e) and $\beta(x) \varepsilon M B(e)=M \alpha(e), \beta^{-1}(x) \varepsilon M \beta^{-1}$ (e) $=M \alpha^{-1}$ (e). Hence $\alpha=\beta$ on Me. Similarly if $\alpha(e)=\beta^{-1}(e)$, we get that $\alpha=\beta^{-1}$ on Me. So we have a central projection e such that $\alpha=\beta$ or $\alpha=\beta^{-1}$ on Me.

So in any case $\alpha^{2}=\beta^{2}$ or $\alpha^{2}=\beta^{-2}$ on Me. Putting $p$ equal to the sum of all such 
central projections $e$, we obtain the required projection $p$ and hence the decomposition of $M$ such that $\alpha^{2}=\beta^{2}$ on $M p$ and $\alpha^{2}=\beta^{-2}$ on $M(1-p)$.

We now come to a general decomposition.

THEOREM 3.2. Let $M$ be a von Neumann algebra and $\alpha, \beta$ be *-automorphisms satisfying: $\alpha+\alpha^{-1}=3+\beta^{-1}$. Then there exists a central projection $p$ in $M$ such that $\alpha^{2}=\beta^{2}$ on Mp and $\alpha^{2}=\beta^{-2}$ on $M(1-p)$.

PROOF. Considering $M$ as a $C^{*}$-algebra, let $\pi_{a}$ be the atomic representation of $M$. Put $N=\overline{\pi_{a}(M)}$ (the $\sigma$-weak closure of $\left.\pi_{a}(M)\right)$. Then $N$ is the direct sum of type I factors. Also the automorphisms $\alpha$ and $\beta$ can uniquely be extended to automorphisms $\tilde{\alpha}$ and $\tilde{\beta}$ of $N$. By continuity $\tilde{\alpha}$ and $\tilde{\beta}$ also satisfy the equation $\tilde{\alpha}+\tilde{\alpha}^{-1}=\tilde{\beta}+\tilde{\beta}^{-1}$. By proposition 3.1, there is a central projection $q$ in $N$ which is invariant under $\tilde{\alpha}$ and $\tilde{\beta}$ and $\tilde{\alpha}^{2}=\tilde{\beta}^{2}$ on $N q$ and $\tilde{\alpha}^{2}=\tilde{\beta}^{-2}$ on $N(1-q)$. Put

and

$$
\begin{aligned}
& I=\left\{x \in M: \pi_{a}(x) q=0\right\} \\
& J=\left\{y \in M: \pi_{a}(y)(1-q)=0\right\} .
\end{aligned}
$$

Then $I$ and $J$ are norm closed ideals of $M$ and also for any $x \in I$ and $y \varepsilon J$,

$$
\begin{aligned}
\pi_{a}(x y) & =\pi_{a}(x) \pi_{a}(y) \\
& =\pi_{a}(x)(1-q) \pi_{a}(y) q \\
& =\pi_{a}(x) \pi_{a}(y) q(1-q)=0 .
\end{aligned}
$$

Thus $I \cdot J=0$. By continuity, it follows that $\bar{I} \cdot \bar{J}=0$ (where $\bar{I}$ denotes the $\sigma$-weak closure of $I$ ). Then there exist central projections $e$ and $f$ in $M$ such that

$$
\bar{I}=M e
$$

and

$$
\bar{J}=\text { Mf and } e \cdot f=0 \text {. }
$$

Since $q$ is fixed under $\tilde{\alpha}$ and $\tilde{\beta}, I$ and $J$ are invariant under $\alpha$ and $\beta$, thus e and $f$ are invariant under $\alpha$ and $\beta$.

Put $I^{\perp}=M(1-e)$ and note that the map $x \rightarrow \pi_{a}(x) q$ is faithful on $I^{\perp}$. Let $y \varepsilon I^{\perp}$. Then both $\alpha^{2}(y)$ and $\beta^{2}(y)$ belong to $I^{\perp}$. Moreover

$$
\begin{aligned}
\pi_{a}\left(\alpha^{2}(y)\right) q=\tilde{\alpha}^{2}\left(\pi_{a}(y)\right) q & =\tilde{\beta}^{2}\left(\pi_{a}(y)\right) q \\
& =\pi_{a}\left(\beta^{2}(y)\right) q .
\end{aligned}
$$

Hence by injectivity of the mapping $x \rightarrow \pi_{a}(x) q$ on $I^{\perp}$, we get that $\alpha^{2}=\beta^{2}$ on $I^{\perp}=M(1-e)$. Similarly $\alpha^{2}=B^{-2}$ on $J^{\perp}=M(1-f)$. As $(1-e)(1-f)=1$, the required decomposition is obtained and the proof is complete.

ACKNOWLEDGEMENT. We are greatly indebted to Professor A. Van Daele for usefu1 comments concerning the counter example in section 2 of this paper. 


\section{REFERENCES}

1. DAELE, A.VAN A new approach to the Tomita-Takesaki theory of generalized Hilbert algebras, J. Func. Analysis 15 (1974), 378-393.

2. THAHEEM, A.B. Decomposition of a von Neumann algebra, Rend. Sem. Mat. Univ. Padova 65 (1981), 1-7.

3. THAHEEM, A.B. and AWAMI, M. A short proof of a decomposition theorem of a von Neumann algebra, Proc. Amer. Math. Soc. (1984), 81-82.

4. THAHEEM, A.B., DAELE, A.VAN and VANHEESWIJEK, L. A result on two one-parameter groups of automorphisms, Math. Scand. 51 (1982), 261-274.

5. THAHEEM, A.B. A bound map associated to a one-parameter group of *-automorphisms of a von Neumann algebra, Glasgow Math. J. 25 (1984), 135-140.

6. CIORANESCU, I. and ZSIDO, L. Analytic generators for one-parameter groups, Tôhoku Math J. 28 (1976), 327-362.

7. HAAGERUP, U. and HANCHE-OLSEN, Tomita-Takesaki theory for Jordan algebras, J. Operator Theory 11 (1984), 343-364.

8. THAHEEM, A.B. On one-parameter of groups of automorphisms of a von Neumann algebra, Proc. of Conf. on Math. Analysis and Its App. (1986), 169-172, Pergamon Press, 1986.

9. DAELE, A.VAN Arvesons' theory of spectral subspaces, Nieuw Arch. Wisk. 27 (1979), 215-237.

10. ARVESON W. On groups of automorphisms of operator algebras, J. Funct. Analysis $15(1974), 217-243$.

11. HAAGERUP, U. and SKAU, C.F. Geometric aspects of the Tomita-Takesaki theory II, Math. Scand. 48 (1981), 241-252.

12. SAKAI, S. Private Communication (1986). 


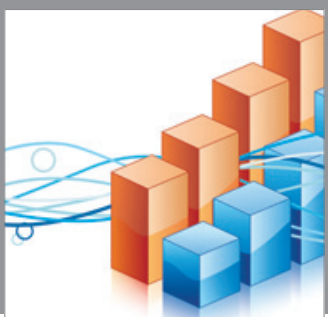

Advances in

Operations Research

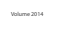

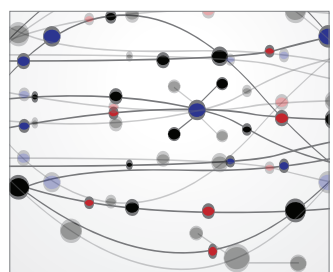

\section{The Scientific} World Journal
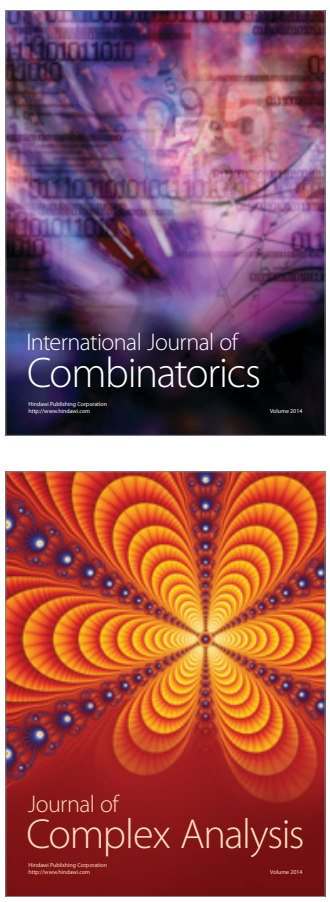

International Journal of

Mathematics and

Mathematical

Sciences
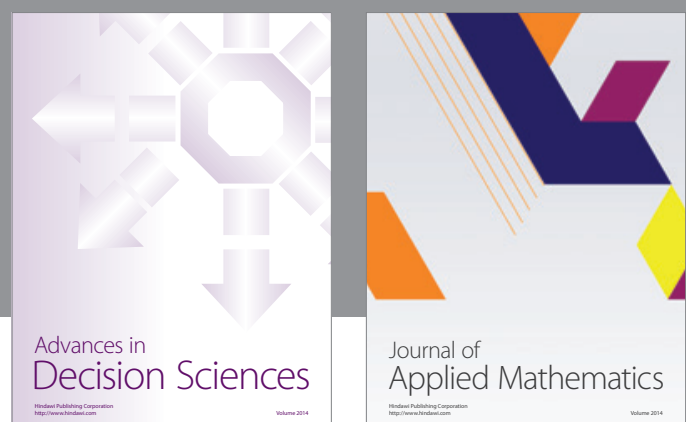

Journal of

Applied Mathematics
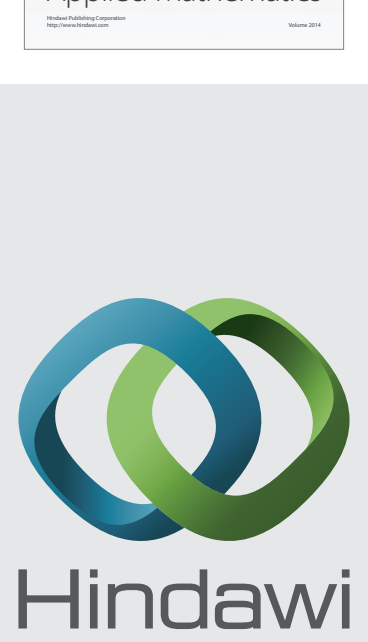

Submit your manuscripts at http://www.hindawi.com
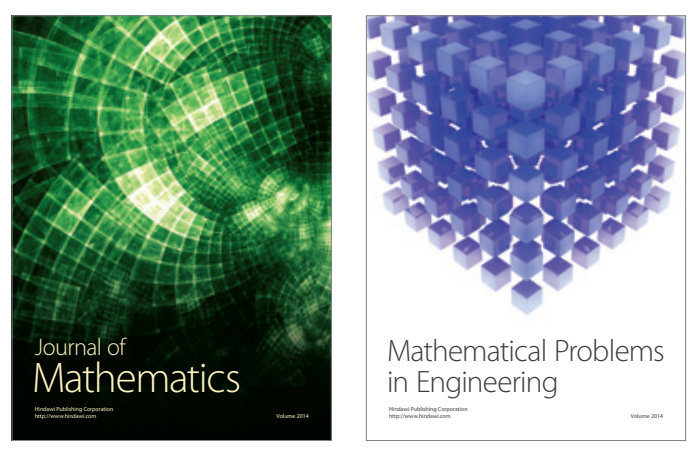

Mathematical Problems in Engineering
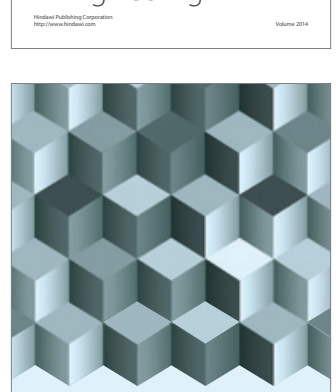

Journal of

Function Spaces
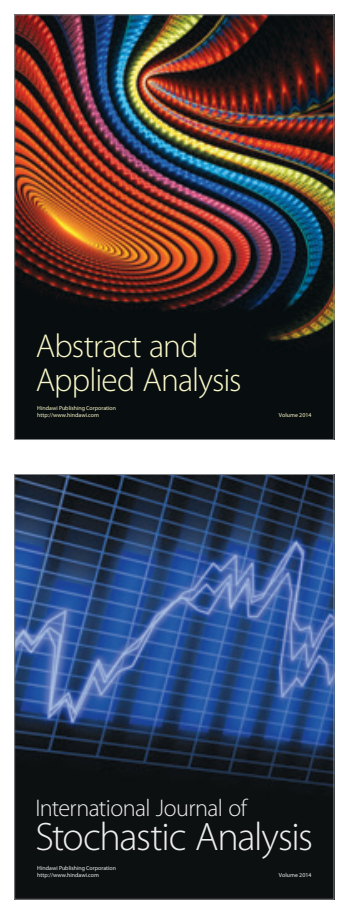

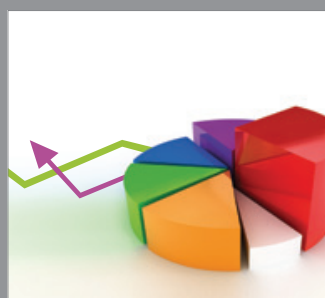

ournal of

Probability and Statistics

Promensencen
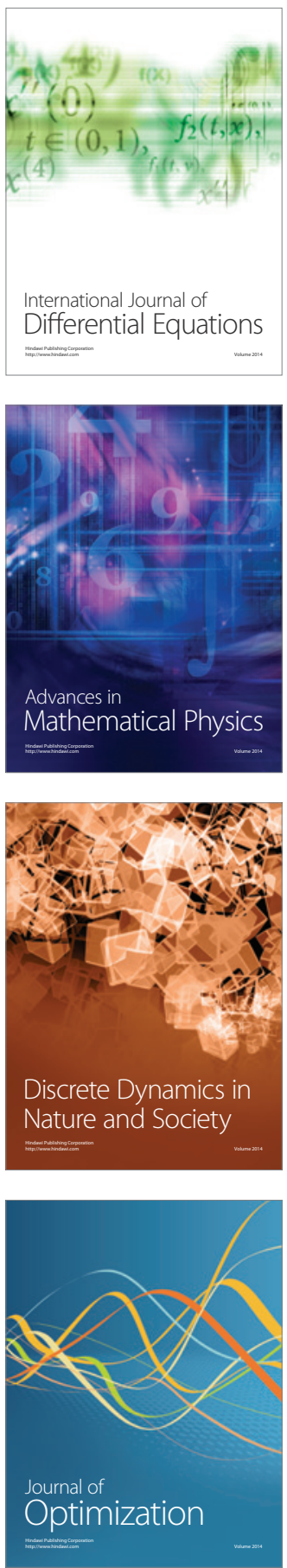